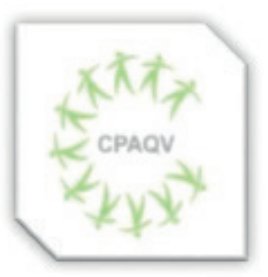

ISSN: $2178-7514$

Vol. 14| No. 1| Ano 2022
ARTIGO DE REVISÃO

\section{EFEITOS DO TREINAMENTO RESISTIDO SOBRE A QUALIDADE DE VIDA DE CRIANÇAS E ADOLESCENTES}

\section{Effects of resistance training on the quality of life of children and adolescents}

Heleise F. R. Oliveira ${ }^{1}$; Guanis B. Vilela Junior ${ }^{1}$; Adriano A. Pereira ${ }^{1}$; Alexandre F. Carvalho ${ }^{13}$; Anderson Martelli ${ }^{4}$; Anderson S. Carvalho ${ }^{1,6}$; Bráulio N. Lima ${ }^{1}$; Gustavo C. Martins ${ }^{1,9,10,11}$; Klebson S. Almeida ${ }^{1,8}$; Luis F. Silio ${ }^{1}$; Marcelo F. Rodrigues ${ }^{1}$; Mariela S. Maneschy ${ }^{8}$; Pedro P. Abdalla ${ }^{1,5}$; Uebister I. S Guedes ${ }^{7}$; Carlos H. P. Fileni ${ }^{1}$; Ricardo P. Passos ${ }^{1,2}$; José Ricardo L. Oliveira ${ }^{1}$

\title{
RESUMO
}

Esta pesquisa abordou os aspectos gerais do treinamento resistido, como; efeitos, possíveis riscos e benefícios que cerceiam o campo da prática da atividade física em crianças e adolescentes, enaltecendo a importância do treinamento resistido para o desenvolvimento corporal e envelhecimento saudável. Por meio de uma revisão de literatura, constatou-se que o treinamento resistido é de suma importância para o desenvolvimento de crianças e adolescentes, tendo em vista que a cada ano essa faixa etária da população tem se tornado cada vez mais sedentária, isso ocorre devido a inúmeros fatores socias, ambientais e psicológicos. Diante do exposto, essa pesquisa traz, como objetivo; compreender como a prática regular do treinamento resistido pode auxiliar na melhora da qualidade de vida de crianças e adolescentes. Como questão problema: como a prática regular do treinamento resistido pode auxiliar na melhora da qualidade de vida de crianças e adolescentes? Desta forma, concluiu-se que o treinamento resistido utilizado como forma de atividade física, de forma assertiva, bem elaborada e supervisionada por profissionais capacitados, pode gerar benefícios a qualidade de vida que vão além do campo fisiológico, promovendo também, bem estar social e mental.

Palavras-chave: Desenvolvimento Infantil. Treinamento Resistido. Exercício Físico.

\begin{abstract}
This research addressed the general aspects of resistance training, such as; effects, possible risks and benefits that limit the field of physical activity in children and adolescents, highlighting the importance of resistance training for body development and healthy aging. Through a literature review, it was found that resistance training is of paramount importance for the development of children and adolescents, given that each year this age group of the population has become increasingly sedentary, this is due to numerous social, environmental and psychological factors. In view of the above, this research has, as its objective; understand how the regular practice of resistance training can help to improve the quality of life of children and adolescents. In this way, it was concluded that resistance training used as a form of physical activity, in an assertive, well-prepared and supervised by trained professionals, can generate benefits that go beyond the physiological field, also promoting social and mental well-being.
\end{abstract}

Keywords: Child Development. Resistance Training. Physical Exercises.

Autor de correspondência

Ricardo Pablo Passos - rppasso@gmail.com

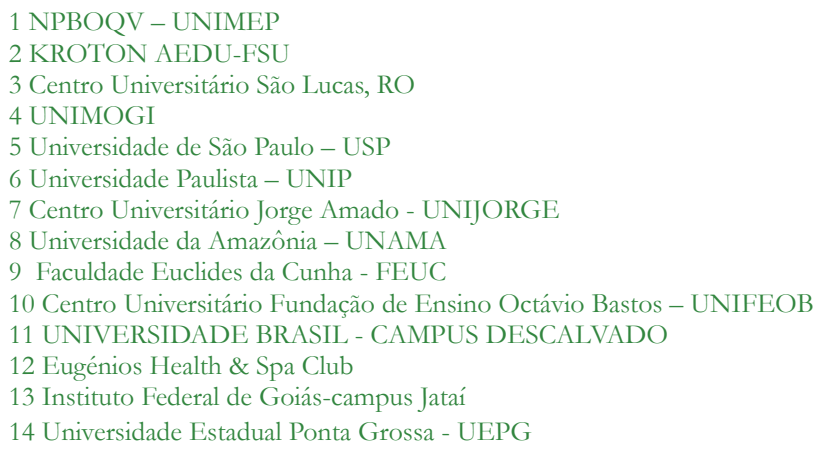




\section{INTRODUÇÃO}

Sabe-se que a atividade física tem uma função fundamental para o desenvolvimento saudável de qualquer indivíduo, proporcionando inegavelmente, benefícios para um funcionamento pleno das funções fisiológicas do corpo humano.

Portanto, quanto mais jovem se inicia a prática da atividade física, menores são os riscos de se desenvolver doenças crônicas como: obesidade; hipertensão; diabetes, entre outras, causadas principalmente pelo sedentarismo.

Atualmente, analisando o cenário epidemiológico do Brasil, a obesidade infantil tem crescido de forma drástica, um dos fatores para esse aumento é a falta de interesse das crianças e adolescentes em atividades físicas, além de hábitos alimentares desequilibrados.

Outro fator avultado que exerce forte influência sobre a inatividade física das crianças e adolescentes nos dias atuais, se dá pelo avanço da tecnologia que proporciona atratividade, fazendo com que a atividade física fique em segundo plano.

Deste modo, se faz necessário incentivar a prática da atividade física para crianças e adolescentes, através de políticas públicas, no contexto escolar, e principalmente em ambiente familiar, gerando assim, um futuro mais saudável e ativo da população.

Dito isso, pode-se considerar que atualmente, existem inúmeras formas de se praticar atividades físicas que proporcionem efeitos benéficos aos praticantes, no entanto, o presente artigo, busca compreender os efeitos e benefícios do treinamento resistido em crianças e adolescentes, porém, indubitavelmente, é importante ressaltar que se faz necessário também, compreender as implicações da prática regular de treinamento resistido, nesse público específico.

Considerando que o treinamento resistido pode oferecer certos riscos aos indivíduos, seja qual for a faixa etária, por se tratar de uma prática executada com pesos de resistência, a presente pesquisa traz a seguinte problematização: como a prática regular do treinamento resistido pode auxiliar na melhora da qualidade de vida de crianças e adolescentes?

Como objetivo, buscou-se compreender como a prática regular do treinamento resistido pode auxiliar na melhora da qualidade de vida de crianças e adolescentes.

\section{MÉTODOS}

Esta pesquisa foi realizada através de uma revisão de literatura, mediante consulta de estudos publicados em livros, artigos científicos e revistas, selecionados nas Bases de Dados: Google Acadêmico; Scielo. Não foram acessados artigos em revistas científicas que cobram pelo acesso ao texto integral dos mesmos. 
Além disso, o período do conteúdo pesquisado foi de 1979 até 2021. Para coletar informações, foram utilizadas as seguintes palavras-chaves: Treinamento Resistido, Exercício Físico, Sedentarismo na Infância, Obesidade Infantil, Atividade Física, Nutrição e qualidade de vida.

Considerações sobre o treinamento resistido, sedentarismo e obesidade em crianças e adolescentes

Para se compreender o conceito de Treinamento Resistido (TR), é de suma importância conhecer a diferença entre atividade física e exercício físico. Atividade física é qualquer movimento locomotor realizado em qualquer contexto, nas tarefas cotidianas, no trabalho, no esporte e nas atividades recreativas fisicamente ativas. Exercício físico se refere ao movimento locomotor intencional, programado e orientado por um profissional de educação física.

Os hábitos de vida saudáveis constituem um aspecto importante em relação a incidência de obesidade em adolescentes pois, além de proporcionarem melhores condições físicas, oferecem benefícios, tais como: diminuição do estresse, adoção de uma melhor cultura alimentar e diminuição do sedentarismo ${ }^{1}$.

Segundo Teixeira ${ }^{2}$ a musculação é a prática de TR mais utilizada na atualidade, despertando o interesse de muitos indivíduos, seja pelos seus benefícios estéticos ou na melhora da saúde, fazendo assim que as academias sejam amplamente procuradas.

Partindo desse pressuposto, o TR pode ser usado como uma ferramenta poderosa na prática de exercícios físicos para crianças e adolescentes.

O TR como o próprio nome ressalta está centrado na realização de atividade física contra uma resistência qualquer. Essa força de resistência pode ser uma massa externa qualquer, com ou sem ajuda de uma máquina, por um segmento corporal ou o próprio peso corporal3.

O engajamento na prática de TR é um instrumento eficiente para ser usado em favor da saúde física, independente da faixa etária dos .praticantes. A prática precoce de atividades físicas em crianças e adolescentes pode proporcionar um estilo de vida futuro mais saudável $^{4}$. Maia e Lopes ${ }^{5}$ definiu através de um estudo que crianças e adolescentes de hoje são fisicamente menos ativos e mais sedentários comparado as crianças e adolescentes dos anos $60,70,80$ e 90 .

A mais recente orientação da Organização Mundial da Saúde6, para essa faixa etária se refere à prática mínima de 60 minutos de atividades físicas diárias, somando 300 minutos por semana. Além de práticas esportivas e atividades lúdicas, como brincadeiras e jogos, que são muito presentes na rotina de crianças e adolescentes, o TR 
também pode ser utilizado como opção de atividade física, desde que, acompanhado de um profissional experiente e capacitado que saiba trabalhar com esse público, respeitando todos os protocolos de segurança.

De acordo com Santarém ${ }^{7}$, a inúmeras discussões entre os estudiosos acadêmicos da área em busca de se obter respostas acerca dos benefícios e malefícios, vantagens e desvantagens da prática de TR em crianças e adolescentes, assim como quais exercícios são mais efetivos e quais protocolos a serem utilizados para proporcionar desenvolvimento e segurança para crianças e adolescentes, respeitando a ludicidade dos mesmos.

Segundo a Associação Americana de Pediatria ${ }^{8}$ o TR garante ganhos na coordenação, flexibilidade e composição corporal, de crianças, sendo, portanto, atividade de relevância para as mesmas.

Rompendo com um mito que o treinamento de força era prejudicial às crianças e adolescentes, nos últimos anos a literatura reporta pesquisas favoráveis à sua utilização no desenvolvimento dos jovens, tendo em vista ser uma atividade segura e reduzido risco de lesões ${ }^{7}$.

Vários estudos têm evidenciado melhoras na composição corporal de jovens que praticam atividade física regular; tal dado, evidencia que a mesma é importante no enfrentamento da epidemia de obesidade que atinge vários países 9 .
Kohl e Hobbs ${ }^{10}$ destacam que o tempo gasto pelas crianças com tecnologias inovadoras (vídeo games, tablets, celulares), não pode ser considerada a causa da redução da atividade física pelos jovens. Trata-se de uma questão complexa que envolve também aspectos sócio econômicos. Nesse sentido, os programas de promoção de saúde destinados aos jovens, devem incluir ações que estimulem a diminuição do tempo de uso diário dessas tecnologias.

Partindo do pressuposto que o sedentarismo contribui para o desenvolvimento de comorbidades, a prevalência da obesidade nas crianças e adolescentes tem aumentado de maneira progressiva e constante, conforme dados levantados pela OMS ${ }^{11}$, na qual mostra que o número de crianças com obesidade era de aproximadamente 44 milhões.

De maneira geral, a obesidade pode ser entendida como uma doença que

provoca acúmulo de gordura no organismo, resultando em um excesso que fica acima dos parâmetros considerados como normais. Esse aumento de gordura traz uma série de fatores que comprometem de forma permanente a saúde de um indivíduo, demandando, assim, a necessidade de se promover estudos para elucidar e promover uma maior conscientização populacional sobre a importância de se ter hábitos alimentares mais saudáveis ${ }^{12,13}$. 
$\mathrm{Na}$ população infantil, o excesso de peso representa cerca de $1 / 5$ do total, fator que está diretamente ligado à promoção de uma geração futura de obesos, pois cerca de $80 \%$ das crianças e adolescentes obesos se tornam adultos com obesidade também. Dentro desse contexto, a obesidade infantil eleva a chance das crianças se tornarem adultos com diversos problemas de saúde advindo pelo excesso de peso, tais como hipertensão, diabetes, riscos renais, entre outras ${ }^{12}$.

\section{Segundo Anjos ${ }^{14}$, a Organização} Mundial da Saúde apontou que em 2025, aproximadamente, se nenhum procedimento for realizado para a redução da obesidade infantil, o número de crianças com sobrepeso e obesidade atingirá a exacerbada marca de 75 milhões, representando uma porcentagem preocupante para a nova geração de adultos.

Além dos problemas físicos, a obesidade infantil também afeta a esfera social das crianças. A obesidade promove dificuldades na interação social, pois elas tendem a descarregar a raiva e demais sentimentos ruins por não serem aceitas em grupos sociais na comida. Assim como a baixa autoestima, o isolamento também pode ser consequência da rejeição por causa da obesidade, contribuindo para o sedentarismo e o isolamento social ${ }^{13}$.

Os benefícios dos exercícios resistidos para essa faixa etária tornam-se mais relevantes pois tem impacto nos custos dos sistemas de saúde, pois jovens obesos tendem a potencializar fatores de risco coronariano, diabetes, hipertensão, dentre outros, com diminuição da percepção da qualidade de vida ${ }^{15}$. Sabendo da importância que o exercício físico exerce sobre a saúde de crianças e adolescentes obesas e sedentárias, é indubitavelmente importante incentivar a prática aos mesmos, seja por orientação dos responsáveis, pela elaboração de políticas públicas ou em âmbito escolar, proporcionando hábitos mais saudáveis e aumentando a qualidade de vida.

\section{Efeitos e benefícios decorrentes da prática de treinamento resistido em crianças e adolescentes}

Conforme exposto na sessão anterior, o exercício físico exerce um papel fundamental na promoção de saúde, seja na infância, adolescência ou na vida adulta. No entanto, é indubitavelmente importante ressaltar que a pratica de exercícios físicos seja rotineira, pois a mesma só exerce influência positiva na saúde quando se é praticada regularmente.

Segundo Bois $^{16}$, a atividade física é importante para condição biopsicossocial das crianças. Em relação aos adolescentes, resultados semelhantes foram obtidos.

O TR traz inúmeros benefícios a saúde corporal, mental e melhora nas relações socias para as crianças e adolescentes, tais como ganho de força provenientes da hipertrofia muscular, 
maior aptidão física e motora resultando em melhor condicionamento físico, menor chance de acometimento a doenças crônicas como a obesidade, aliado na melhora do rendimento em esportes evitando também as lesões, realização e satisfação devido ao desenvolvimento pessoal e o reconhecimento social, além de benefícios psicológicos relativos ao humor e ao aumento de confiança.

O treinamento físico melhora da capacidade respiratória, resultado do treino com frequência, promovendo adaptações na capacidade cardiorrespiratória e na capacidade ventilatória de crianças e adolescentes ${ }^{7}$.

Segundo Quevedo ${ }^{17}$ o TR pode ser utilizado como ferramenta na reabilitação e prevenção de lesões devido ao aumento de força muscular, proporcionando também melhor funcionalidade do aparelho neuro locomotor, com incremento da potência e melhor controle postural.

Complementando os benefícios descritos acima, estudos evidenciam ainda mais a capacidade de efeitos benéficos oriundos do TR, como melhora no perfil lipídico devido ao aumento do metabolismo e consequentemente a queima de gordura corporal, melhor desempenho das funções cardiovasculares, redução de fatores de riscos e comorbidades. Constatou-se também que a prática de TR em crianças e adolescentes promove benefícios recreativos, e melhor desempenho em atividades diárias e escolares18. Tais resultados culminam com o aumento do número e nível de ativação de neurônios no sistema nervoso, assim a prática de exercícios físicos contribui com a capacidade de concentração, da fixação de conteúdo, desenvolvimento do raciocínio lógico e da memória ${ }^{17}$.

\section{Considerações sobre estágios da} infância e adolescência para a prática de exercícios físicos

Algumas considerações são importantes para que se possa compreender qual o melhor momento de se iniciar a prática de exercícios físicos em crianças e adolescentes.

Segundo Piaget (1960), na evolução cognitiva das crianças por volta dos 11/12 anos, que elas têm condições de participar de um plano sistemático de exercícios físicos, orientando-se corretamente e julgando desempenhos e competências. Para o American College of Sports Medicine (1995) a faixa etária dos 6 a 12 anos é quando ocorre a consolidação do repertório de habilidades das crianças.

Quanto mais adiantado for o grau de maturação do adolescente, maior será o nível de testosterona depois da prática de exercícios físicos, o que resulta em melhor capacidade de realizar força após a puberdade. No transcorrer da infância e no início da adolescência, não existem diferenças significantes na capacidade de realizar força entre ambos os sexos, porém, após esse período os rapazes gradualmente 
ficam mais fortes, fruto das diferenças hormonais ${ }^{18}$.

\section{Promoção da prática de exercícios}

\section{físicos na infância e adolescência}

Diante do exposto no tópico anterior, fica evidente a importância que a promoção da atividade física exerce em crianças e adolescentes, pois é uma estratégia importante para evitar o surgimento de doenças crônicas não transmissíveis. Assim, a prática de atividade física aliada à alimentação adequada é essencial para que a criança fisicamente ativa garanta seu potencial de crescimento e desenvolvimento e tenha seu desempenho elevado. Entretanto, para que os benefícios da atividade física tenham reflexos em um estilo de vida saudável, é fundamental que esta seja praticada de forma regular e consistente, pois os benefícios são temporários ${ }^{19}$.

Para que fatores de riscos como a obesidade na infância e adolescência seja controlada e deixe de ser um problema de saúde pública, é importante que ocorra um processo de Educação em Saúde de qualidade e assertivo, voltado para a educação populacional sobre a pratica de exercícios físicos e a importância de se alimentar corretamente ${ }^{20}$.

Hohepa $^{21}$ pesquisou a percepção de adolescentes sobre os diversos contextos ligados à atividade física e estratégias para promoção da atividade física. Como resultado os jovens reportaram aspectos que se referem ao acesso, suporte da família e motivação pessoal, como características importantes

Partindo desse pressuposto, se faz necessário estratégias que possibilitem o desenvolvimento de um estilo de vida saudável não apenas pelos próprios indivíduos, mais também por influência e fatores externos, como apoio familiar, possibilitando assim, a promoção de atividades e exercícios físicos no público alvo desse estudo.

\section{Implicações e riscos a prática} de treinamento resistido em crianças e adolescentes

Algumas implicações de naturezas diversas relacionadas a prática rotineira de exercícios físicos na infância e adolescência foram apontadas nos estudos realizados.

Segundo Hohepa ${ }^{21}$, afirma que fatores como falta de tempo, baixo nível de motivação, ambientes que estimulam o sedentarismo, baixa estrutura ligada a prática de exercícios físicos, influência adversa dos amigos e obstáculos físicos, afetam diretamente a prática de exercícios físicos na infância e adolescência.

Outro fator de peso que implica na prática de exercícios físicos na infância e juventude, é o prazer pelo mesmo, caso as atividades não proporcionarem satisfação ou conforto para a criança, certamente ela irá desistir de praticá-la, todavia, se ocorrer 
o contrário, ela certamente maximizará os benefícios.

Além das barreiras citadas acima, a uma grande preocupação dos pais em relação aos possíveis efeitos negativos que o exercício físico pode gerar na criança e no adolescente propriamente dito. A aplicação da musculação para crianças tem sido motivo de controvérsias, principalmente pela falta de conhecimento, por parte do educador físico, de vários aspectos relacionados ao crescimento da criança.

Fleck $^{22}$, reporta que essa faixa etária pode ter problemas crônicos e agudos na coluna lombar devido à sobrecarga oriunda do treinamento. Isso reforça a importância de um profissional de educação física para a prescrição do exercício físico.

Gordia et al. ${ }^{23}$, destacam que as crianças apresentam menor débito

de suor porglândula quando comparados aos adultos e, portanto, ficam expostas durante o exercício ao estresse térmico, colocandoas em risco de hipertermia. Isso determina a importância da hidratação adequada.

Segundo Weineck ${ }^{24}$, os ossos dos jovens, em razão da contínua sedimentação, não são compactos, sendo mais sensíveis, razão pela qual se deve evitar uma sobrecarga.

As preocupações acerca de eventuais riscos que o TR pode acarretar em crianças e adolescentes, tem sido substituída por evidencias científicas recentes.
Treinamento resistido com pesos para crianças e adolescentes

O foco do programa de TR em crianças e adolescentes deve ser o desenvolvimento da flexibilidade das articulações, incrementar a força dos tendões antes da força dos músculos e aumentar a força central antes da força dos membros ${ }^{25}$.

O Colégio Americano de Medicina Esportiva $^{18}$ e Marques $^{26}$, sugerem que um programa de treinamento para crianças e adolescentes iniciantes deve ser ter duração de 20 a 60 minutos por sessão, três dias da semana, sendo inicialmente com exercícios de baixa intensidade até que a técnica do movimento esteja consolidada.

Para Ughini et $\mathrm{al}^{27}$ os modelos de treinamento para essa faixa etária podem ser os mesmos aplicados a grupos adultos; no entanto, as crianças não devem ser consideradas como adultos em miniatura, dessa forma, os pressupostos e filosofias de treino dos mais velhos, não devem ser impostos aos adolescentes.

De acordo com Fleck e Kraeme ${ }^{3}$, os seguintes parâmetros para a progressão de programa de treino para crianças e adolescentes devem ser preconizados:

a) 5 a 7 anos: realizar exercícios básicos com pouco ou nenhuma carga, utilizando, preferencialmente o peso do 
próprio corpo ou de um parceiro, mas sempre com cargas leves e volume baixo;

b) $\quad 8$ a 10 anos: aumentar a quantidade de exercícios e, gradualmente, a carga, realizando exercícios simples, com aumento lento do volume e monitoramento da tolerância ao estresse físico;

c) 11 a 13 anos: introduzir o ensino das técnicas, com aumento gradual do peso, realização de exercícios mais avançados com pouca ou nenhuma carga;

d) 14 a 15 anos: realizar exercícios de força mais avançados, com ênfase

na técnica, e aumentar o volume de treino;

e) 16 anos em diante: aplicar programa destinado a pessoas adultas;

Para que essa proposta tenha êxito é importante haja a atuação de um profissional de educação física no processo, pois o mesmo é habilitado e capacitado para realizar os procedimentos necessários como, prévia avaliação física do aluno, aplicação de anamnese, identificação de fatores de risco, entre outros protocolos, e assim, garantir a segurança da aplicabilidade de exercícios físicos como o TR em crianças e adolescentes.

\section{CONSIDERAÇÕES FINAIS}

De acordo com as pesquisas que foram utilizadas para o desenvolvimento desse estudo, observou-se que o sedentarismo tem feito cada vez mais parte na rotina das crianças e adolescentes, pois a atratividade fornecida pela tecnologia avançada como vídeo games e mídias sociais, tem ocupado o tempo que antes era destinado as atividades e exercícios físicos. Como consequência negativa a esse fato, a obesidade tem aumentado drasticamente nesse público, trazendo riscos à saúde dos jovens. Dessa maneira, ficou evidente a extrema importância do exercício físico na rotina das crianças e adolescentes para reverter esse quadro.

De maneira geral, o treinamento resistido utilizado como alternativa a prática de exercícios físico em crianças e adolescentes proporciona inúmeros benefícios a saúde dos praticantes, melhorando o perfil lipídico e diminuindo riscos de comorbidades, maximizando o desempenho em várias atividades rotineiras como estudos escolares e prática de esportes, podendo colaborar nos aspectos sociais e psicológicos.

Diante do exposto referente aos benefícios gerados pelo treinamento resistido, faz-se necessário políticas públicas que promovam, incentivem e proporcionem oportunidades a prática de exercícios físicos na infância e adolescência, sendo extremamente 
necessário também o apoio familiar ao jovem

referente hábitos de vida saudáveis.

No entanto, algumas orientações são de extrema relevância no tocante a prática de treinamento resistido com jovens, sendo necessário o acompanhamento profissional para que eventuais lesões sejam evitadas. Como toda prática de exercício físico deve ser bem orientada e prescrita por profissionais da área, seja qual for a faixa etária.

\section{REFERÊNCIAS}

1- GOLDENER, L.J. (2013). Educação física e saúde: benefício da atividade física para saúde e qualidade de vida (Trabalho de conclusão de curso). UFES. Acesso em 17 abr. 2021.

2- TEIXEIRA, Cauê V. La Scala. Musculação para crianças. Disponível em: www.educacaofisica.org/ wp/?p=1467. Acesso em 22 mar. 2021.

3- KRAEMER, J. William; FLECK, J. Steven. Otimizando o treinamento de força: programas de periodização não linear. Barueri, SP: Manole, p.195, 2009.

4- SILVA, P.G. Carolina; BITTAR, M. L. Cléria. Fatores Ambientais e psicológicos que influenciam na obesidade infantil. Revista Saúde e Pesquisa, v.5, n.1, p.197-207, 2012.

5- ALVES, C., LIMA, R.V.B. (2008). Impacto da atividade física e esportes sobre o crescimento e puberdade de crianças e adolescentes. Revista Paulista de Pediatria, 26(4). 383-91. Acesso em 06 mar. 2021.

6- OMS- World Health Organization. Global strategy on diet, physical activity and health: childhood overweight and obesity. Relatório encontrado na internet. 2014.

7- SANTAREM, José Maria. Musculação em todas as idades: Comece a praticar antes o seu médico recomende. Barueri. Manole. 2012

8- Academia Americana de Pediatria. (2008). Treinamento de Força por Crianças e Adolescentes. Conselho de Medicina Esportiva e Fitness, 121(4). 835840. Disponível em:http://pediatrics.aappublications. org/content/pediatrics/121/4/835.full.pdf Acesso em 18 mar. 2021.
9- SHAIBI, G.Q., CRUZ, M.L., BOLA, G.D., WEIGENSBERG, M.J., SALEM, G.J., CRESPO, N.C., GORAN, M.I.(2006). Efeitos do treinamento de resistência na sensibilidade à insulina em adolescentes latinos com excesso de peso. Medicina e Ciência em Esportes e Exercícios, 38 (7): 1208-1215. Acesso em 23 mar. 2021

10- KOHL, H. W., \& HOBBS, K. E. (1998). Development of physical activity behaviors among children and adolescents. Pediatrics, 101(3 Pt 2), 554- 569.

11- AMERICAN COLLEGE OF SPORTS MEDICINE (1995). Position stand on quantity and quality of exercise for developing and maintaining cardiorespiratory, musculoskeletal, and neuromotor fitness in apparently healthy adults: guidance for prescribing exercise. Medicine and Science in Sports and Exercise. Vol. 43. n.7. 2011. Disponível em: https://www.ncbi.nlm.nih. gov/pubmed/21694556. Acesso em 22 mar. 2021.

12- ASCARI, R. A. et al. Obesidade Infantil: um olhar dos enfermeiros inseridos na atenção básica. $17^{\circ}$ seminário Nacional de Pesquisa em Enfermagem. 2013. Disponível em: http:/ / www.abeneventos.com.br/anais_ senpe/17senpe/pdf/0627co.pdf. Acesso em 02 mar. 2021.

13- ROCHA, L. M. da. Obesidade Infantil: uma revisão bibliográfica. Dissertação de Especialização apresentada à Universidade Federal de Minas Gerais. 2013. Disponível em: https://www.nescon.medicina.ufmg.br/biblioteca/ imagem/6400.pdf. Acesso em 02 abr. 2021

14- ANJOS, L. A. D. Obesidade e saúde. $2^{\text {a }}$ ed. Rio de Janeiro: Fiocruz, 2016. Biddle, S. J. H., Gorely, T., \& Stensel, D. J. (2004). Healthenhancing physical activity and sedentary behavior in children and adolescents. Journal of Sports Sciences, 22(8), 679-701.

15- QUADROS, T.M.B, GORDIA, A.P., MOTA, J., BARROS, M.V.G., GUIMARÃES, I., AZEVEDO, H., GUEDES, P. (2017). Promoção da Atividade Física na Infância e Adolescência. Sociedade Brasileira de Pediatria, 1. Acesso em 17 abr. 2021

16- BOIS, J. E., SARRAZIN, P. G., BRUSTAD, R. J., TROUILLOUD, D. O., \& CURY, F. (2005). Elementary schoolchildren's perceived competence and physical activity involvement: The influence of parents' role modeling behaviors and perceptions of their child's competence. Psychology of Sport and Exercise, 6(4), 381-397.

17- QUEVEDO, Juliana Villate. Passagem da infância para a adolescência, 2013. Disponível em: https://www. aberta.org.br/educarede/2013/05/21/passagem-dainfancia-para-a- adolescencia/ Acesso em: 07 abr. 2021.

18- AMERICAN COLLEGE OF SPORTS MEDICE (ACSM) (2007). Diretrizes do ACSM para os testes de esforço e sua prescrição (7a ed.). Rio de Janeiro: Guanabara Koogan.

19- BRACCO, Mário Maia et al. Atividade física na 
infância e adolescência: impacto na saúde pública. Revista de Ciências Médicas, Campinas, v. 12, n.1, p. 8997, jan./mar., 2003. Disponível em: <http://periodicos. puc- campinas.edu.br/seer/index.php/cienciasmedicas / article/view/1283 Acesso em 05 abr. 2021

20- FREITAS, A. S. S. et al. Obesidade infantil: influência de hábitos alimentares inadequados. Saúde e Ambiente. V. 4, n. 2, p. 9-14, 2009. Disponível em: http://www. educadores.diaadia.pr.gov.br/arquivos/File/2010/ artigos_teses/2010/ Biolo gia/artigos/obesoinfantil. pdf. Acesso em 16 abr. 2021.

21- HOHEPA, M., SCHOFIELD, G., \& KOLT, G. S. (2006). Physical activity: What do high school students think? Journal of Adolescent Health, 39(3), 328-336.

22- FLECK, S. J., KRAEMER, W. J. (2017). Fundamentos do treinamento de força muscular (4a ed.). Porto Alegre: Artmed.

23- GORDIA, A.P., QUADROS, T.M.B., SILVA, L.R., SANTOS, G.M. (2015).

Conhecimento de pediatras sobre a atividade física na infância e adolescência. Revista Paulista de Pediatria 33(4). 400-406. Disponível em: http://www.scielo.br/ pdf/rpp/v33n4/pt_0103-0582-rpp-33-04- 0400.pdf. doi: http://dx.doi.org/10.1016/j.rpped.2015.02.001. Acesso em 07 abr. 2021.

24- WEINECK, J. (2005). Biologia do Esporte (7a ed.). São Paulo: Manole.

25- COSTA, A., PINHEIRO, V., CIPRIANO, M., SEQUEIRA, P. (2008). O Treino de Força no período Infanto-Juvenil: Algumas considerações Pedagógicas e Metodológicas. Revista de Desporto e Atividade Física, 1 (2), 1-19. Disponível em: http://www. juventudevidigalense.pt/pdf/artigos / 2016/O\%20 Treino $\% 20 \mathrm{de}^{\%} \mathrm{20 \text {For }}$

$\%$ C3\%A7a \%20com\%20Jovens.pdf. Acesso em 07 abr. 2021.

26- MARQUES, M.A.C. (2010). O treino de força em crianças e jovens. Revista Medicina in forma, 1(6). 21-24. Disponível em: http://revdesportiva. pt/files/6Novembro2010/site_treino_da_forca_ MarioMarq.pd

f. Acesso em 23 abr. 2021.

27- UGHINI, C.C., BECKER, C., PINTO, R.S. (2011). Treinamento de força em crianças: segurança, benefícios e recomendações. Conexões, educação física, esportes e saúde, 9(2). 177-197. Disponível em: file:///C:/Users/ Nathalia/Downloads/8637706-7768-2-PB.pdf. Acesso em 06 abr. 2021

OBSERVAÇÃO: Os autores declaram não existir conflitos de interesse de qualquer natureza. 